\section{PELVIC INFLAMMATORY DISEASE (PID), MYCOPLASMA GENITALIUM AND MACROLIDE RESISTANCE IN ENGLAND}

${ }^{1}$ Gillian Dean, ${ }^{1}$ Jennifer Whetham, ${ }^{1}$ Suneeta Soni, ${ }^{2}$ Rachel Pitt*, ${ }^{2}$ Sarah Alexander. ${ }^{1}$ Brighton \& Sussex University Hospitals NHS Trust, Brighton, UK; ${ }^{2}$ Sexually transmitted bacteria reference unit (STBRU), PHE, Colindale, London, UK

\subsection{6/sextrans-2016-052718.30}

Background/introduction Mycoplasma genitalium (MG) is increasingly implicated in PID pathogenesis with many studies showing MG is as common as chlamydia in high-risk women. Current PID treatment guidelines specify antibiotics with low efficacy against MG. Increasing reports of macrolide resistance suggests first line treatment for MG (azithromycin) may have limitations.

Aim(s)/objectives To document rates of MG in a cohort of women with acute PID, and the proportion with baseline macrolide resistance.

Methods As part of a multicentre, open-label, non-inferiority RCT comparing ofloxacin/metronidazole (arm-1) with azithromycin $1 \mathrm{~g}$ day-1; $500 \mathrm{mg}$ od day 2-5, metronidazole/ceftriaxone (arm-2), samples were collected for baseline chlamydia, gonorrhoea and mycoplasma infection. Microbiological cure rates were documented at 6-8 weeks. Positive MG specimens were examined for macrolide resistance using a 23S rRNA PCR.

Results 313 women were recruited, median age 25. Preliminary results showed chlamydia was confirmed in $9.5 \%$, MG in $8.2 \%$ and gonorrhoea in $0.4 \%$. Of the 16 samples available for resistance testing, 9 (56\%) had macrolide resistance mutations (A2058G/T, A2059G/C) at baseline. The reference laboratory received test-of-cure samples for only 8 patients with MG, of which 6 were negative, however 2 remained positive, both with A2059G nucleotide substitutions. Further results will be presented.

Discussion/conclusion MG infection was nearly as common as chlamydia in this cohort. Failure of patients to return at 6-8 weeks affected our ability to properly assess test-of-cure rates. Baseline macrolide resistance was unexpectedly high and impacted negatively on treatment success.

\section{IMPLEMENTING A TEST AND TREAT PATHWAY FOR MYCOPLASMA GENITALIUM IN MEN WITH URETHRITIS ATTENDING A GUM CLINIC}

John Reynolds-Wright*, Fabienne Verrall, Mohammed Hassan-Ibrahim, Suneeta Soni. Brighton and Sussex University Hospitals NHS Trust, Brighton, UK

\subsection{6/sextrans-2016-052718.31}

Background/introduction National guidelines recommend testing men with non-gonococcal urethritis(NGU) for Mycoplasma geni$\operatorname{talium}(\mathrm{MG})$ where testing is available. Recent studies have shown concerning levels of macrolide resistance and high rates of treatment failure with $1 \mathrm{~g}$ azithromycin. In response to this, we changed our standard treatment of NGU to doxycycline and implemented a test and treat pathway for MG in male NGU.

Aim(s)/objectives To determine the prevalence of $M G$ and to measure clearance rates of infection post-treatment.

Methods From $1^{\text {st }}$ September 2015 first void urine samples from men with NGU were routinely tested for MG using the Fast Track Diagnostics $^{\mathrm{TM}}$ urethritis PCR. Men with confirmed MG were given 5 days azithromycin and offered test of cure at 4 weeks.
Results 304 men had NGU over 5months. Mean age 33.2 years, $64.5 \%$ heterosexual, $77.6 \%$ white ethnicity and $8.2 \%$ HIV-positive. Tests for MG were performed in 230/304 (76\%). 16.5\% (38/230) were MG positive compared with $22.5 \%$ chlamydia. 6 (15.8\%) men with MG were co-infected with chlamydia and 1 (2.6\%) had urethral gonorrhoea. 20/38 men with MG $(52.6 \%)$ were given azithromycin 5 days, $8 / 38$ (21.1\%) had azithromycin $1 \mathrm{~g}$ stat. $7 / 38(18.4 \%)$ re-tested positive at 4 weeks, 6 of whom had initially had azithromycin 5 days. All were given moxifloxacin 400mg for either 7,10 or 14 days.

Discussion/conclusion The high rates of MG found support routine testing in men with NGU. Despite appropriate treatment, some men returned with positive TOC suggestive of macrolide failure. This demonstrates a need for more widely available MG diagnostics with resistance testing in the UK and attention to antimicrobial stewardship so that NGU can be better managed.

\section{A QPCR ASSAY THAT SIMULTANEOUSLY DETECTS MYCOPLASMA GENITALIUM AND MUTATIONS ASSOCIATED WITH MACROLIDE RESISTANCE HAS THE POTENTIAL TO IMPROVE PATIENT MANAGEMENT}

${ }^{1}$ Terry Sunderland*, ${ }^{1}$ Lit Yeen Tan, ${ }^{1}$ Samantha Walker, ${ }^{2}$ Jenny Su, ${ }^{3}$ Catriona Bradshaw, ${ }^{3}$ Chris Fairley, ${ }^{2}$ Suzanne Garland, 'Elisa Mokany, ${ }^{2}$ Sepehr Tabrizi. 'SpeeDx Pty Ltd, Sydney, NSW, Australia; ${ }^{2}$ The Royal Children's and The Royal Women's Hospitals, Melbourne, VIC, Australia; ${ }^{3}$ Melbourne Sexual Health Centre, Melbourne, VIC, Australia

\subsection{6/sextrans-2016-052718.32}

Background Treatment of M. genitalium $(\mathrm{Mg})$ infection with azithromycin, is routinely utilised in clinical practice. However, widespread use has been associated with the emergence of macrolide resistance and ineffective cure rates. A new qPCR assay, PlexPCR $^{\mathrm{TM}}$ M. genitalium ResistancePlus ${ }^{\mathrm{TM}}$ kit, has been developed to simultaneously identify $\mathrm{Mg}$ and 5 mutations in the $23 \mathrm{~S}$ rRNA gene (positions 2058 and 2059 (E. coli numbering)) associated with macrolide resistance.

Aim This study evaluates incorporating the assay into a diagnostic algorithm to direct faster and more appropriate clinical management and reduce the spread of antibiotic resistant.

Methods 1087 consecutive urogenital samples from symptomatic and asymptomatic patients were evaluated prospectively with the PlexPCR M. genitalium ResistancePlus kit. This was compared to an in-house test for $\mathrm{Mg}$ detection and sequencing of $\mathrm{Mg}$ positives to determine 23S rRNA mutation status. The PlexPCR $M$. genitalium ResistancePlus kit employs novel PlexPrime (amplifies mutants specifically) and PlexZyme (superior multiplexing) technology.

Results The prevalence of $\mathrm{Mg}$ was $6.0 \%$ and in the $\mathrm{Mg}$ positive samples $23 \mathrm{~S}$ rRNA mutation prevalence was $63.1 \%$. The PlexPCR M. genitalium ResistancePlus assay showed very high clinical performance compared to the reference methods with sensitivity and specificity for $\mathrm{Mg}$ detection of $98.5 \%$ and $100.0 \%$, and $23 \mathrm{~S}$ rRNA mutation detection of $92.7 \%$ and 95.7\% respectively.

Conclusion The PlexPCR M. genitalium ResistancePlus kit demonstrated excellent clinical performance for the simultaneous detection of $\mathrm{Mg}$ and assessment of macrolide resistance. This test has the potential to be used in screening of $\mathrm{Mg}$ detection and macrolide resistance to allow more appropriate clinical management. 